\title{
The neural mechanisms for the recognition of face identity in humans
}

\author{
Stefano Anzellotti ${ }^{1,2}$ * and Alfonso Caramazza ${ }^{1,2}$ \\ ' Department of Psychology, Harvard University, Cambridge, MA, USA \\ ${ }^{2}$ Center for Mind/Brain Sciences, University of Trento, Trento, Italy
}

\section{Edited by:}

Chris Fields, New Mexico State

University, USA (retired)

\section{Reviewed by:}

Aude Oliva, Massachusetts Institute

of Technology, USA

Chris Fields, New Mexico State

University, USA (retired)

${ }^{*}$ Correspondence:

Stefano Anzellotti, Department of Psychology, Harvard University,

William James Hall, 33 Kirkland

Street, Cambridge, MA 02138, USA

e-mail: anzellot@fas.harvard.edu
Every day we encounter dozens of people, and in order to interact with them appropriately we need to recognize their identity. The face is a crucial source of information to recognize a person's identity. However, recognizing the identity of a face is challenging because it requires distinguishing between very similar images (e.g., the front views of two different faces) while categorizing very different images (e.g., a front view and a profile) as the same person. Neuroimaging has the whole-brain coverage needed to investigate where representations of face identity are encoded, but it is limited in terms of spatial and temporal resolution. In this article, we review recent neuroimaging research that attempted to investigate the representation of face identity, the challenges it faces, and the proposed solutions, to conclude that given the current state of the evidence the right anterior temporal lobe is the most promising candidate region for the representation of face identity.

\section{Keywords: faces, identity, fMRI, object recognition, invariance}

\section{INTRODUCTION}

In this paper, we focus on recent neuroimaging research that has investigated aspects of the neural mechanisms underlying the perceptual recognition of face identity. The ability to recognize individuals is crucial for guiding behavior - it allows us to retrieve information about people and interact with them in appropriate ways. Many different cues can be used to recognize an individual, including the appearance of the face, the sound of the voice, as well as the context in which we encounter a person and prior knowledge about his/her current general location (see Oliva and Torralba, 2007; Goesaert and Op de Beeck, 2013). A promising approach consists in studying how each of these cues is processed when other cues are controlled, to then proceed with an investigation of how the different cues are integrated. Among the different cues that can be used for person recognition, the face is a crucial source of information and is usually sufficient in isolation to recognize a person's identity. However, recognizing face identity is also computationally challenging: it requires discounting identity-irrelevant changes in sensory stimulation (such as changes in viewpoint and illumination) without losing the ability to perform fine-grained discriminations needed to distinguish the faces of similar individuals.

The earliest insights into the neural mechanisms underlying the ability to recognize face identity came from the study of patients with selective impairment for the recognition of faces (Charcot, 1883; Wilbrand, 1892; Heidenhain, 1927; Jossmann, 1929), which was subsequently named prosopagnosia (Bodamer, 1947). Hecaen and Angelergues (1962) investigated the location of lesions producing selective deficits for faces in a group of 22 patients, and observed that prosopagnosic patients tended to have lesions in the right hemisphere, often involving occipital regions. A review of the neuropsychological literature individuated the right occipitotemporal cortex as the most common location of the lesion in prosopagnosic patients (Meadows, 1974). Convergent evidence in support of the view that damage to the occipitotemporal cortex leads to prosopagnosia was reported in several studies (Whiteley and Warrington, 1977; Damasio et al., 1982; Malone et al., 1982).

Other neuropsychological studies reported deficits for the recognition of familiar and famous faces in patients with herpes simplex encephalitis (Warrington and Shallice, 1984; Warrington and McCarthy, 1988) and semantic dementia (Snowden et al., 2004), with more frequent face recognition deficits in the right than in the left temporal variant of semantic dementia (Thompson et al., 2003). These pathologies affect the anterior portions of the temporal lobe (Kapur et al., 1994; Mummery et al., 2000; Gitelman et al., 2001; Hodges and Patterson, 2007; Noppeney et al., 2007). Furthermore, the highest lesion overlap in patients with face recognition deficits was found the be in the right anterior temporal lobe (Tranel et al., 1997). Consistent with the neuropsychological literature, neuroimaging studies in healthy participants individuated regions showing stronger activity for faces than for other kinds of objects in occipitotemporal cortex [occipital face area (OFA) and fusiform face area (FFA); Sergent et al., 1992; Puce et al., 1996; Kanwisher et al., 1997; Gauthier et al., 2000; see Çukur et al., 2013 for an in-depth analysis of voxel response profiles] and the anterior temporal lobes (Rajimehr et al., 2009).

Both occipitotemporal regions and anterior temporal regions show stronger activity for faces than other objects, and lesions in these regions lead to face processing deficits. What are the respective contributions of the two brain regions in representing face identity? The finding that lesion to a brain region leads to a deficit for face recognition does not imply that that region encodes representations of face identity - it might just provide necessary input to another region that represents face identity. At the same time, neither occipitotemporal nor anterior temporal regions seem to be involved merely in the processing of "low level" 
perceptual details. Patients with anterior temporal lesions have intact basic perceptual abilities (Warrington and Shallice, 1984), and while patients with occipitotemporal lesions often have visual field defects (Meadows, 1974), they are able to describe and draw individual face parts (Bodamer, 1947). A deeper understanding of the properties of representations in these regions is needed to clarify their respective roles for the recognition of face identity. This paper is concerned with the neuroimaging research pursuing this understanding. In particular, the focus is on perceptual representations of face identity, rather than on other aspects of person identity such as associated semantic knowledge (Tsukiura et al., 2002), or the sense of familiarity and emotional responses which can be impaired in disorders such as Capgras syndrome (Ellis and Lewis, 2001).

\section{DISCRIMINATION OF FACE TOKENS}

Before delving into the discussion of the literature, it is necessary to introduce some terms and clarify their use. We will use the term "face token" to refer to a specific image of a face, seen from a particular viewpoint and under a particular illumination. The recognition of face identity requires (1) to distinguish between face tokens that depict different people, and (2) to recognize when two different face tokens depict the same person. We will use the term "invariant face representations" to refer to representations that encode information about whether two face tokens depict the same person, for some or all pairs of face tokens that depict a same person. Note that invariance can be partial, for example, there might be representations that are invariant to changes in viewpoint of up to $35^{\circ}$. Therefore, not all invariant face representations are representations of face identity. We will reserve the term "representation of face identity" for representations that encode information that allows determining that two face tokens depict the same person for all pairs of face tokens that are recognized as a same person by a human observer. Whether or not there exists one brain region that encodes representations with invariance across all transformations that humans can generalize across is an empirical question. To search for representations of face identity, we can first search for representations that distinguish between face tokens that depict different people, and then test whether and to which extent they are invariant. Finding brain regions that distinguish between face tokens that depict different people provides us with a series of potential candidates for the representation of face identity.

The investigation of regions that distinguish between face tokens that depict different people with functional magnetic resonance imaging (fMRI) is challenging, because when properties like viewpoint and illumination are controlled, face tokens that depict different people do not produce significantly different blood-oxygen-level dependent (BOLD) responses when analyzed with standard univariate approaches. Nonetheless, fMRI remains one of the best methods available to localize regions that distinguish between face tokens that depict different people. This is because it allows coverage of a large extent of the human brain in a single study, and because among the methods with this property it is the one that offers the highest spatial resolution.
For this reason, in the course of the past two decades, researchers used fMRI to investigate the neural mechanisms underlying the recognition of face identity, developing and employing experimental designs and data analysis approaches to meet the challenge posed by the subtle differences in the BOLD responses produced by different face tokens.

One approach to individuating representations that distinguish between face tokens that depict different people involves using fMRI-adaptation ( $\mathrm{fMR}-\mathrm{A}$ ). FMR-A is a phenomenon characterized by reduced BOLD responses to repeated stimuli (Grill-Spector et al., 1999). FMR-A has also been observed during the presentation of two stimuli that are not identical but are similar along some dimension (Grill-Spector et al., 1999; Vuilleumier et al., 2002). For example, fMR-A can occur for the presentation of different stimuli from the same category (Fairhall et al., 2011). FMR-A has been used to investigate representations of face tokens in a series of studies (Grill-Spector et al., 1999; Gauthier et al., 2000; Rotshtein et al., 2004; Furl et al., 2007). Greater adaptation for repetitions of a same face token than for the presentation of different face tokens has been observed in the FFA (Gauthier et al., 2000), as well as in occipitotemporal regions defined with a broader contrast between faces and textures (Grill-Spector et al., 1999).

As an alternative to fMR-A, some researchers have used multivariate pattern analysis (MVPA) to improve the sensitivity of fMRI (Haxby etal., 2001; Haynes and Rees, 2006). Multivariate approaches extract information from the pattern of activity in multiple voxels. They are more sensitive than univariate approaches, because they can distinguish between BOLD responses within a region that have the same mean but different spatial distributions.

A common method consists in using univariate analyses in order to individuate regions showing stronger responses to faces than other objects ("face-selective" regions) and subsequently investigate information content with MVPA within these regions. With this regions-of-interest (ROI) approach it has been shown that face-selective regions, including notably the FFA, encode information about face tokens (Nestor et al., 2011; Anzellotti et al., 2013; Goesaert and Op de Beeck, 2013; Verosky et al., 2013; but see Natu etal., 2010). However, this approach is based on the implicit assumption that localizing the brain regions showing the greatest mean difference between the activity in response to faces and the activity in response to other objects exhaustively captures the regions involved in the recognition of face identity. This assumption might not hold: there may be regions that do not show face-selectivity but still contribute to the recognition of face identity.

An alternative to the use of face selectivity is searchlight analysis (Kriegeskorte et al., 2006; Kriegeskorte and Bandettini, 2007) to individuate regions that distinguish between face tokens in the whole brain. In an early study (Kriegeskorte et al., 2007), searchlight was used to detect information that distinguishes between face tokens in the right anterior temporal lobe. The faces that were distinguished, though, were of different genders. A more recent study (Nestor et al., 2011) used searchlight and individuated information that distinguishes between face tokens of the same gender in the right anterior temporal lobe and posterior temporal cortex bilaterally. 
Another method that can be used to individuate information that distinguishes between face tokens is recursive feature elimination (RFE), a type of MVPA (De Martino et al., 2008; Formisano et al., 2008). RFE has advantages (and some disadvantages) with respect to both ROI-based and searchlight methods. RFE can individuate information that is distributed beyond the extent of a searchlight sphere. It does not require that a set of contiguous voxels classify the different conditions significantly above chance; that is, informative voxels can be anywhere in the brain. This also means that feature selection approaches do not require making arbitrary choices about the size and shape of the regions within which to search for information. In addition, RFE requires that the individuated voxels contribute themselves to the discrimination, while in the case of searchlight an individuated voxel does not necessarily contribute to the discrimination: as long as other voxels within the sphere provide significant classification accuracy, the voxel will appear in the searchlight map, even if the voxel itself is not informative (this is especially true for SVMbased searchlight, see Etzel et al., 2013). The main disadvantage of RFE is that in its current form it allows localization of voxels that contribute to a given classification, but unlike searchlight and representational similarity analysis (RSA) it does not allow localization of regions based on a match between a neural dissimilarity matrix and a dissimilarity matrix hypothesized by the experimenter. However, for the purpose of localization of regions involved in the representation of face tokens this is not a major concern. To date, RFE has produced promising results for the localization of regions that distinguish between face tokens that depict different people (Figure 1), allowing localization of informative voxels for the discrimination between gender-matched faces in occipitotemporal and anterior temporal regions (Nestor et al., 2011; Anzellotti et al., 2013), and in the posterior cingulate and the posterior intraparietal sulcus (Anzellotti and Caramazza, 2014).

In sum, regions that distinguish between face tokens that depict different people have been found in occipitotemporal cortex bilaterally, in the anterior temporal lobes, in posterior cingulate and in bilateral IPS. Very recent studies (Cowen et al., 2014; Nestor et al., 2014) adopted principal component analysis (PCA) and independent component analysis (ICA) to investigate classification for larger numbers of face tokens, going beyond the small number of identities used in most studies to date.

\section{INVARIANT FACE REPRESENTATIONS}

Regions that distinguish between face tokens that depict different people are candidate regions for representing face identity, but not all of them necessarily encode representations of face identity. To individuate regions that represent face identity, it is important to investigate whether they encode invariant face representations. Studies investigating the invariance of face representations typically look for evidence of commonalities among representations of different face tokens that depict the same person. For this reason, it is particularly important to carefully control the stimuli used because the presence of commonalities in the low-level properties of different face tokens depicting a same person can lead to illusory invariance effects. Equating the average luminance, color and texture in the whole image is often insufficient as a control because visually responsive neurons at several stages of processing have local receptive fields that do not encompass the entire image. These challenges can be overcome by generating stimuli with computer graphics. Using computer graphics permits the careful control of the low-level

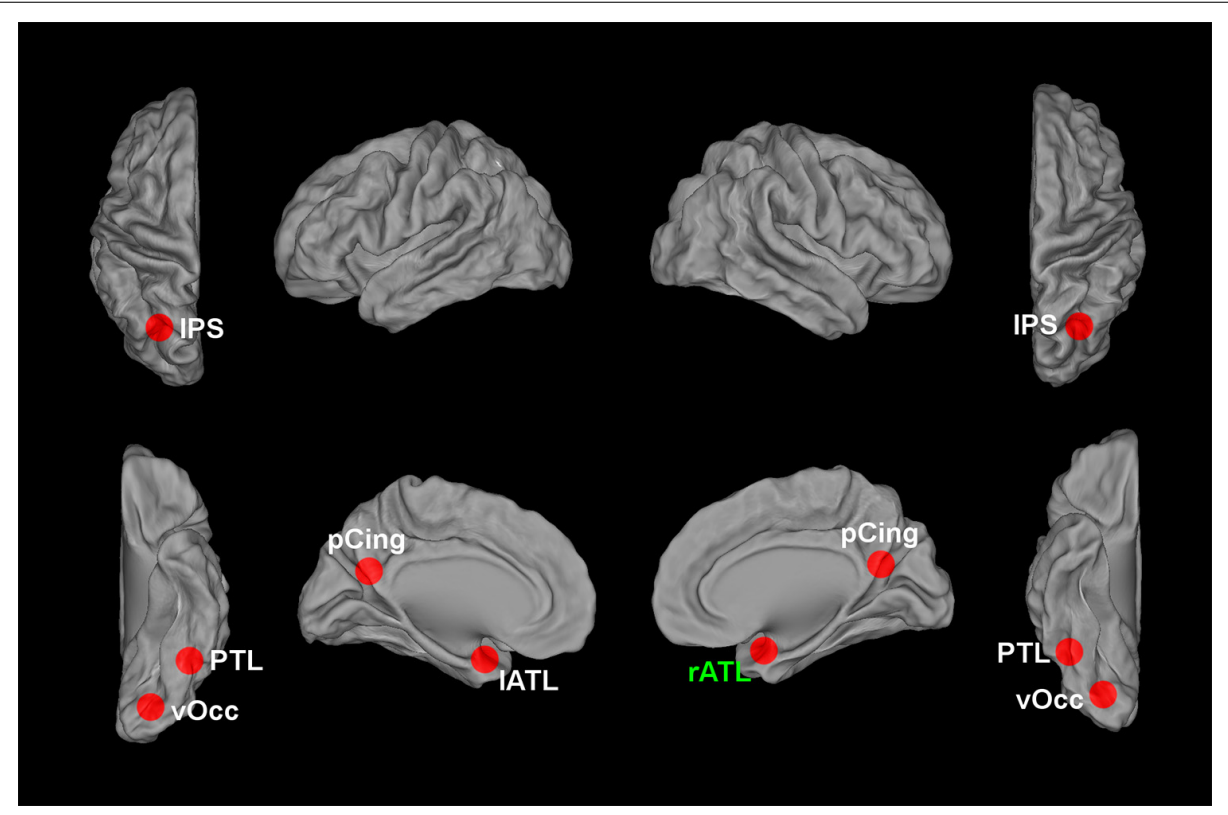

FIGURE 1 | Brain regions encoding information that contributes to the classification between different face tokens corresponding to different individuals. $v O c c$, ventral occipital cortex; PTL, posterior temporal lobe; ATL, anterior temporal lobe; pCing, posterior cingulate; IPS, intraparietal sulcus. The current evidence indicates the right ATL, marked in green, as the most likely candidate region for encoding invariant representations of face identity. 
differences between face tokens at a local level (Anzellotti et al., 2013; Anzellotti and Caramazza, 2014). Since even cartoon faces elicit strong responses in face-selective neurons (Freiwald etal., 2009), it is unlikely that the use of realistic 3D renderings of faces would bias the results with respect to the use of photographs.

fMRI-adaptation can be used not only to individuate regions sensitive to differences in identity, but also to search for commonalities among representations of different face tokens that depict a same person. If a region encodes invariant face representations, the representations of different face tokens depicting the same person should overlap more than the representations of face tokens depicting different people, and therefore more fMR-A should be observed for the presentation of different face tokens that depict a same person than face tokens of different persons. One problem with the underlying assumptions motivating the use of fMR-A to study invariant face representations is that even if we accept that regions encoding invariant face representations should show fMR-A for the presentation of different face tokens depicting a same person, it does not follow that all regions that show fMRA for the presentation of different face tokens depicting a same person encode invariant face representations. One way in which a region could show fMR-A for different face tokens depicting a same person despite encoding non-invariant face representations is through top-down influences. Via top-down influences, recognition of two different images as tokens depicting a same identity could lead to reduced activity not only in regions encoding invariant representations but also in early visual regions. Whether or not reduction in neural activity due to repetition can occur as a consequence of top-down influences is controversial (Xiang and Brown, 1998; Schendan and Kutas, 2003).

Several studies investigated invariant face representations using fMR-A, with mixed results: some studies found evidence for adaptation (Vuilleumier et al., 2002) while others did not (Pourtois et al., 2005). Ewbank and Andrews (2008) found fMR-A for repetition of face identity across different viewpoints in FFA when presenting familiar faces, but not when presenting novel faces. The likelihood of observing adaptation across different face tokens depicting a same person in fMR-A studies seems to be a function of the duration of the lag between two stimuli, with longer lags leading to more invariance in some studies (Andresen et al., 2009), but it remains unclear what are the mechanisms at the basis of this phenomenon. A recent study (Mur et al., 2010) found fMR-A for the repetition of face identity across different viewpoints in several regions, including early visual cortex. Given the current understanding of representations in early visual cortex, it is unlikely that this region carries invariant face representations. Findings such as this suggest that fMR-A can occur due to top-down influences.

To overcome the interpretative challenges that arise in fMRA studies, invariant face representations have been investigated with MVPA. Experiments designed to investigate invariance with MVPA typically involve the presentation of multiple different tokens (e.g., different facial expressions, different viewpoints) of each face identity. The BOLD responses to those face tokens are then split into a subset used for the training of a classifier (for instance a support vector machine), and a subset used for the testing of the performance of the trained classifier. A possible approach is to split the data into subsets so that each part contains responses to all stimuli shown. In this case, the training and testing subsets contain the BOLD signal in response to different presentations of the same identical images. This analysis approach is not circular (data from different runs are used for the training and testing of classifiers), but since responses to the same images are used for training and testing, the classifier could potentially achieve significant classification accuracy relying on representations that are not invariant.

Despite these remarks, a recent study (Nestor et al., 2011) used this approach and found accuracies significantly above chance in FFA but at chance in early visual cortex for the classification of face identity in the presence of different facial expressions (Nestor et al., 2011). The robust classification accuracies obtained in this study (Nestor et al., 2011) are probably due to the contribution of invariant representations. However, other studies reported significant classification accuracy for faces seen from different viewpoints even in early visual cortex when using this method (Anzellotti et al., 2013). This is in contrast with the current understanding of representations in early visual cortex, and suggests that the conclusions obtained with this method should be interpreted with caution.

A more stringent method that overcomes the concerns discussed above consists in splitting the data into subsets so that the responses to different viewing conditions are included in the training and the testing set. In this case, the training and testing subsets contain the BOLD signal in response to different images. Using this method, classification across different viewpoints was at chance in early visual cortex, but was significant in other ventral stream regions (Anzellotti et al., 2013). In particular, even when using the responses to different stimuli for training and testing, and controlling carefully the "low-level" properties of images, significant classification generalizing across viewpoints was observed in both occipitotemporal and anterior temporal regions (Anzellotti et al., 2013). However, significant classification does not directly imply that a region carries representations of identity. The extent to which representations are invariant to transformations may vary, and a brain region could show invariance for some image transformations that humans can generalize across, but not for others. According to our definitions, such a representation would count as an invariant representation, but not as a representation of face identity.

Individuating significant classification accuracy across some specific transformations in multiple brain regions does not imply that the regions encode the same kind of representations. Therefore, occipitotemporal regions and anterior temporal regions might still encode different representations. To test this, a recent experiment investigated whether representations in different brain regions encoded information about face identity generalizing across different face halves (Anzellotti and Caramazza, 2014). For this manipulation, invariance was only found in the right anterior temporal lobe, and not in occipitotemporal cortex.

In the process of generating increasingly invariant representations, some information about identity-irrelevant differences between face tokens might be discarded or represented implicitly (DiCarlo and Cox, 2007). For this reason, the study of how and where identity-irrelevant information (e.g., information about viewpoint, illumination, and so on) is encoded can be seen as 
a complementary investigation to the study of invariance. Several studies provide evidence that identity-irrelevant information declines moving from posterior to anterior regions in the ventral stream (Kietzmann et al., 2012; Anzellotti and Caramazza, 2014; see Freiwald and Tsao, 2010 for similar evidence in monkeys, and Yovel and Freiwald, 2013 for a discussion of issues of homology). However, some identity-irrelevant information might still be present in more anterior regions (DiCarlo and Maunsell, 2003; Kravitz et al., 2008).

\section{CONCLUSION}

Investigating the neural mechanisms underlying the recognition of face identity in humans is challenging, but the continuous development and improvement of design and analysis techniques has allowed the localization of representations that distinguish between face tokens depicting different people, and to begin to investigate their invariance. Given the current state of neuroimaging evidence, one region seems to encode face representations showing greatest invariance: the right anterior temporal lobe (Anzellotti et al., 2013; Anzellotti and Caramazza, 2014). This conclusion is consistent with neuropsychological evidence of deficits for face recognition after damage to the right anterior temporal lobe (Tranel et al., 1997), and with electrophysiology studies in monkeys (Freiwald and Tsao, 2010). However, it is important to note that current evidence does not establish that the right anterior temporal lobe is the only locus of face identity recognition: bilateral deficits are frequent in the anterior temporal lobes, and thus it remains possible that the left anterior temporal lobe also contributes, although to a lesser extent, to the recognition of face identity. In previous studies, the anterior temporal lobes have been implicated in semantic knowledge (Hodges et al., 1992; Tsukiura et al., 2002; Patterson et al., 2007). Invariant face representations could play an important role to link perceptual inputs to semantic knowledge about people.

Invariance does not appear only in the anterior temporal lobe, but builds up gradually, being present already to some extent in occipitotemporal regions (Kietzmann et al., 2012; Anzellotti et al., 2013; see Freiwald and Tsao, 2010 for consistent electrophysiology findings in monkeys), suggesting different roles for occipitotemporal and anterior temporal cortex for the recognition of face identity.

\section{ACKNOWLEDGMENTS}

Stefano Anzellotti was supported by a dissertation completion fellowship from Harvard University, Alfonso Caramazza's research was supported in part by the Fondazione Cassa di Risparmio di Trento e Rovereto.

\section{REFERENCES}

Andresen, D. R., Vinberg, J., and Grill-Spector, K. (2009). The representation of object viewpoint in human visual cortex. Neuroimage 45, 522-536. doi: 10.1016/j.neuroimage.2008.11.009

Anzellotti, S., and Caramazza, A. (2014). "Invariant representations of face identity in the ATL," in Beyond the FFA: The role of the Ventral Anterior Temporal Lobes in Face Processing. Symposium Conducted at the Annual Meeting of the Vision Sciences Society, eds J. Collins and I. Olson (Chairs), St. Pete Beach, FL.

Anzellotti, S., Fairhall, S. L., and Caramazza, A. (2013). Decoding representations of face identity that are tolerant to rotation. Cereb. Cortex doi: 10.1093/cercor/bht046 [Epub ahead of print].
Bodamer, J. (1947). Die Prosop-Agnosie. Arch. Psychiatr. Nervenkr. 179, 6-53. doi: 10.1007/BF00352849

Charcot, J. M. (1883). Lectures on the Localisation of Cerebral and Spinal Diseases, Vol. 102. London: New Sydenham Society, 178-179.

Cowen, A. S., Chun, M. M., and Kuhl, B. A. (2014). Neural portraits of perception: reconstructing face images from evoked brain activity. Neuroimage 94, 12-22. doi: 10.1016/j.neuroimage.2014.03.018

Çukur, T., Huth, A. G., Nishimoto, S., and Gallant, J. L. (2013). Functional subdomains within human FFA. J. Neurosci. 33, 16748-16766. doi: 10.1523/JNEUROSCI.1259-13.2013

Damasio, A. R., Damasio, H., and Van Hoesen, G. W. (1982). Prosopagnosia: anatomic basis and behavioral mechanisms. Neurology 32, 331-331. doi: 10.1212/WNL.32.4.331

De Martino, F., Valente, G., Staeren, N., Ashburner, J., Goebel, R., and Formisano, E. (2008). Combining multivariate voxel selection and support vector machines for mapping and classification of fMRI spatial patterns. Neuroimage 43, 44-58. doi: 10.1016/j.neuroimage.2008.06.037

DiCarlo, J. J., and Cox, D. D. (2007). Untangling invariant object recognition. Trends Cogn. Sci. 11, 333-341. doi: 10.1016/j.tics.2007.06.010

DiCarlo, J. J., and Maunsell, J. H. (2003). Anterior inferotemporal neurons of monkeys engaged in object recognition can be highly sensitive to object retinal position. J. Neurophysiol. 89, 3264-3278. doi: 10.1152/jn.00358.2002

Ellis, H. D., and Lewis, M. B. (2001). Capgras delusion: a window on face recognition. Trends Cogn. Sci. 5, 149-156. doi: 10.1016/S1364-6613(00)01620-X

Etzel, J. A., Zacks, J. M., and Braver, T. S. (2013). Searchlight analysis: promise, pitfalls, and potential. Neuroimage 78, 261-269. doi: 10.1016/j.neuroimage.2013.03.041

Ewbank, M. P., and Andrews, T. J. (2008). Differential sensitivity for viewpoint between familiar and unfamiliar faces in human visual cortex. Neuroimage 40, 1857-1870. doi: 10.1016/j.neuroimage.2008.01.049

Fairhall, S. L., Anzellotti, S., Pajtas, P. E., and Caramazza, A. (2011). Concordance between perceptual and categorical repetition effects in the ventral visual stream. J. Neurophysiol. 106, 398-408. doi: 10.1152/jn.01138.2010

Formisano, E., De Martino, F., Bonte, M., and Goebel, R. (2008). "Who" is saying "what"? Brain-based decoding of human voice and speech. Science 322, 970-973. doi: 10.1126/science.1164318

Freiwald, W. A., and Tsao, D. Y. (2010). Functional compartmentalization and viewpoint generalization within the macaque face-processing system. Science 330, 845-851. doi: 10.1126/science. 1194908

Freiwald, W. A., Tsao, D. Y., and Livingstone, M. S. (2009). A face feature space in the macaque temporal lobe. Nat. Neurosci. 12, 1187-1196. doi: 10.1038/nn.2363

Furl, N., van Rijsbergen, N. J., Treves, A., and Dolan, R. J. (2007). Face adaptation aftereffects reveal anterior medial temporal cortex role in high level category representation. Neuroimage 37, 300-310. doi: 10.1016/j.neuroimage.2007. 04.057

Gauthier, I., Tarr, M. J., Moylan, J., Skudlarski, P., Gore, J. C., and Anderson, A. W. (2000). The fusiform "face area" is part of a network that processes faces at the individual level. J. Cogn. Neurosci. 12, 495-504. doi: 10.1162/089892900562165

Gitelman, D. R., Ashburner, J., Friston, K. J., Tyler, L. K., and Price, C. J. (2001). Voxelbased morphometry of herpes simplex encephalitis. Neuroimage 13, 623-631. doi: 10.1006/nimg.2000.0734

Goesaert, E., and Op de Beeck, H. P. (2013). Representations of facial identity information in the ventral visual stream investigated with multivoxel pattern analyses. J. Neurosci. 33, 8549-8558. doi: 10.1523/JNEUROSCI. 1829-12.2013

Grill-Spector, K., Kushnir, T., Edelman, S., Avidan, G., Itzchak, Y., and Malach, R. (1999). Differential processing of objects under various viewing conditions in the human lateral occipital complex. Neuron 24, 187-203. doi: 10.1016/S08966273(00)80832-6

Haxby, J. V., Gobbini, M. I., Furey, M. L., Ishai, A., Schouten, J. L., and Pietrini, P. (2001). Distributed and overlapping representations of faces and objects in ventral temporal cortex. Science 293, 2425-2430. doi: 10.1126/science.1063736

Haynes, J. D., and Rees, G. (2006). Decoding mental states from brain activity in humans. Nat. Rev. Neurosci. 7, 523-534. doi: 10.1038/nrn1931

Hecaen, H., and Angelergues, R. (1962). Agnosia for faces (prosopagnosia). Arch. Neurol. 7, 92-100. doi: 10.1001/archneur.1962.04210020014002

Heidenhain, D. A. (1927). Beitrag zur kenntnis der seelenblindheit. Eur. Neurol. 66, 61-74. doi: 10.1159/000165960 
Hodges, J. R., and Patterson, K. (2007). Semantic dementia: a unique clinicopathological syndrome. Lancet Neurol. 6, 1004-1014. doi: 10.1016/S14744422(07)70266-1

Hodges, J. R., Patterson, K., Oxbury, S., and Funnell, E. (1992). Semantic dementia progressive fluent aphasia with temporal lobe atrophy. Brain 115, 1783-1806. doi: 10.1093/brain/115.6.1783

Jossmann, P. (1929). Zur Psychopathologie der optisch-agnostischen Storungen, Mschr. Psychiatr. Neurol. 77, 81-149. doi: 10.1159/000164409

Kanwisher, N., McDermott, J., and Chun, M. M. (1997). The fusiform face area: a module in human extrastriate cortex specialized for face perception. J. Neurosci. $17,4302-4311$.

Kapur, N., Barker, S., Burrows, E. H., Ellison, D., Brice, J., Illis, L. S., et al. (1994) Herpes simplex encephalitis: long term magnetic resonance imaging and neuropsychological profile. J. Neurol. Neurosurg. Psychiatry 57, 1334-1342. doi: 10.1136/jnnp.57.11.1334

Kietzmann, T. C., Swisher, J. D., König, P., and Tong, F. (2012). Prevalence of selectivity for mirror-symmetric views of faces in the ventral and dorsal visual pathways. J. Neurosci. 32, 11763-11772. doi: 10.1523/JNEUROSCI.0126-12.2012

Kravitz, D. J., Vinson, L. D., and Baker, C. I. (2008). How position dependent is visual object recognition? Trends Cogn. Sci. 12, 114-122. doi: 10.1016/j.tics.2007.12.006

Kriegeskorte, N., and Bandettini, P. (2007). Combining the tools: activationand information-based fMRI analysis. Neuroimage 38, 666-668. doi: 10.1016/j.neuroimage.2007.06.030

Kriegeskorte, N., Formisano, E., Sorger, B., and Goebel, R. (2007). Individual faces elicit distinct response patterns in human anterior temporal cortex. Proc. Natl. Acad. Sci. U.S.A. 104, 20600-20605. doi: 10.1073/pnas.0705654104

Kriegeskorte, N., Goebel, R., and Bandettini, P. (2006). Information-based functional brain mapping. Proc. Natl. Acad. Sci. U.S.A. 103, 3863-3868. doi: 10.1073/pnas.0600244103

Malone, D. R., Morris, H. H., Kay, M. C., and Levin, H. S. (1982). Prosopagnosia: a double dissociation between the recognition of familiar and unfamiliar faces. J. Neurol. Neurosurg. Psychiatry 45, 820-822. doi: 10.1136/jnnp.45.9.820

Meadows, J. C. (1974). Disturbed perception of colours associated with localized cerebral lesions. Brain 97, 615-632. doi: 10.1093/brain/97.1.615

Mummery, C. J., Patterson, K., Price, C. J., Ashburner, J., Frackowiak, R. S. J., and Hodges, J. R. (2000). A voxel-based morphometry study of semantic dementia: relationship between temporal lobe atrophy and semantic memory. Ann. Neurol. 47, 36-45. doi: 10.1002/1531-8249(200001)47:1<36:AID-ANA8>3.0.CO;2-L

Mur, M., Ruff, D. A., Bodurka, J., Bandettini, P. A., and Kriegeskorte, N. (2010). Face-identity change activation outside the face system: "release from adaptation" may not always indicate neuronal selectivity. Cereb. Cortex 20, 2027-2042. doi: 10.1093/cercor/bhp272

Natu, V. S., Jiang, F., Narvekar, A., Keshvari, S., Blanz, V., and O’Toole, A. J. (2010). Dissociable neural patterns of facial identity across changes in viewpoint. J. Cogn. Neurosci. 22, 1570-1582. doi: 10.1162/jocn.2009.21312

Nestor, A., Plaut, D. C., and Behrmann, M. (2011). Unraveling the distributed neural code of facial identity through spatiotemporal pattern analysis. Proc. Natl. Acad. Sci. U.S.A. 108, 9998-10003. doi: 10.1073/pnas.1102433108

Nestor, A., Plaut, D. C., and Behrmann, M. (2014). "Facial identity: an investigation of neural encoding and image reconstruction," in Face Perception: Neural Mechanisms. Talk Session Conducted at the Annual Meeting of the Vision Sciences Society, St. Pete Beach, FL.

Noppeney, U., Patterson, K., Tyler, L. K., Moss, H., Stamatakis, E. A., Bright, P., et al. (2007). Temporal lobe lesions and semantic impairment: a comparison of herpes simplex virus encephalitis and semantic dementia. Brain 130, 1138-1147. doi: 10.1093/brain/awl344

Oliva, A., and Torralba, A. (2007). The role of context in object recognition. Trends Cogn. Sci. 11, 520-527. doi: 10.1016/j.tics.2007.09.009

Patterson, K., Nestor, P. J., and Rogers, T. T. (2007). Where do you know what you know? The representation of semantic knowledge in the human brain. Nat. Rev. Neurosci. 8, 976-987. doi: 10.1038/nrn2277

Pourtois, G., Dan, E. S., Grandjean, D., Sander, D., and Vuilleumier, P. (2005). Enhanced extrastriate visual response to bandpass spatial frequency filtered fearful faces: time course and topographic evoked - potentials mapping. Hum. Brain Mapp. 26, 65-79. doi: 10.1002/hbm.20130
Puce, A., Allison, T., Asgari, M., Gore, J. C., and McCarthy, G. (1996). Differential sensitivity of human visual cortex to faces, letterstrings, and textures: a functional magnetic resonance imaging study. J. Neurosci. 16, 5205-5215.

Rajimehr, R., Young, J. C., and Tootell, R. B. (2009). An anterior temporal face patch in human cortex, predicted by macaque maps. Proc. Natl. Acad. Sci. U.S.A. 106, 1995-2000. doi: 10.1073/pnas.0807304106

Rotshtein, P., Henson, R. N., Treves, A., Driver, J., and Dolan, R. J. (2004). Morphing Marilyn into Maggie dissociates physical and identity face representations in the brain. Nat. Neurosci. 8, 107-113. doi: 10.1038/nn1370

Schendan, H. E., and Kutas, M. (2003). Time course of processes and representations supporting visual object identification and memory. J. Cogn. Neurosci. 15, 111135. doi: 10.1162/089892903321107864

Sergent, J., Ohta, S., and Macdonald, B. (1992). Functional neuroanatomy of face and object processing: a positron emission tomography study. Brain 115, 15-36. doi: 10.1093/brain/115.1.15

Snowden, J. S., Thompson, J. C., and Neary, D. (2004). Knowledge of famous faces and names in semantic dementia. Brain 127, 860-872. doi: 10.1093/brain/awh099

Thompson, S. A., Patterson, K., and Hodges, J. R. (2003). Left/right asymmetry of atrophy in semantic dementia: behavioral-cognitive implications. Neurology 61, 1196-1203. doi: 10.1212/01.WNL.0000091868.28557.B8

Tranel, D., Damasio, H., and Damasio, A. R. (1997). A neural basis for the retrieval of conceptual knowledge. Neuropsychologia 35, 1319-1327. doi: 10.1016/S00283932(97)00085-7

Tsukiura, T., Fujii, T., Fukatsu, R., Otsuki, T., Okuda, J., Umetsu, A., et al. (2002). Neural basis of the retrieval of people's names: evidence from brain-damaged patients and fMRI. J. Cogn. Neurosci. 14, 922-937. doi: $10.1162 / 089892902760191144$

Verosky, S. C., Todorov, A., and Turk-Browne, N. B. (2013). Representations of individuals in ventral temporal cortex defined by faces and biographies. Neuropsychologia 51, 2100-2108. doi: 10.1016/j.neuropsychologia.2013. 07.006

Vuilleumier, P., Henson, R. N., Driver, J., and Dolan, R. J. (2002). Multiple levels of visual object constancy revealed by event-related fMRI of repetition priming. Nat. Neurosci. 5, 491-499. doi: 10.1038/nn839

Warrington, E. K., and McCarthy, R. A. (1988). The fractionation of retrograde amnesia. Brain Cogn. 7, 184-200. doi: 10.1016/0278-2626(88)90029-2

Warrington, E. K., and Shallice, T. (1984). Category specific semantic impairments. Brain 107, 829-853. doi: 10.1093/brain/107.3.829

Whiteley, A. M., and Warrington, E. K. (1977). Prosopagnosia: a clinical, psychological, and anatomical study of three patients. J. Neurol. Neurosurg. Psychiatry 40, 395-403. doi: 10.1136/jnnp.40.4.395

Wilbrand, H. (1892). Ein Fall von Seelenblindheit und Hemianopsie mit Sectionsbefund. Dtsch. Z. Nervenheilkd. 2, 361-387. doi: 10.1007/BF01667704

Xiang, J. Z., and Brown, M. W. (1998). Differential neuronal encoding of novelty, familiarity and recency in regions of the anterior temporal lobe. Neuropharmacology 37, 657-676. doi: 10.1016/S0028-3908(98) 00030-6

Yovel, G., and Freiwald, W. A. (2013). Face recognition systems in monkey and human: are they the same thing? F1000prime Rep. 5, 10. doi: 10.12703/P5-10

Conflict of Interest Statement: The authors declare that the research was conducted in the absence of any commercial or financial relationships that could be construed as a potential conflict of interest.

Received: 14 February 2014; accepted: 10 June 2014; published online: 26 June 2014. Citation: Anzellotti S and Caramazza A (2014) The neural mechanisms for the recognition of face identity in humans. Front. Psychol. 5:672. doi: 10.3389/fpsyg.2014.00672 This article was submitted to Perception Science, a section of the journal Frontiers in Psychology.

Copyright (c) 2014 Anzellotti and Caramazza. This is an open-access article distributed under the terms of the Creative Commons Attribution License (CC BY). The use, distribution or reproduction in other forums is permitted, provided the original author(s) or licensor are credited and that the original publication in this journal is cited, in accordance with accepted academic practice. No use, distribution or reproduction is permitted which does not comply with these terms. 